\title{
Interdisciplinary and transferable concepts in bioinformatics education: observations and approaches from a UK MSc course
}

\author{
lain G. Johnston ${ }^{1,2, *}$, Mark Slater ${ }^{3}$, Jean-Baptiste Cazier ${ }^{4,5}$ \\ ${ }^{1}$ Department of Mathematics, Faculty of Mathematics and Natural Sciences, University of Bergen, Norway \\ 2 Computational Biology Unit, University of Bergen, Norway \\ ${ }^{3}$ School of Physics and Astronomy, College of Engineering and Physical Science, University of Birmingham, UK \\ 4 Institute of Cancer and Genomic Sciences, University of Birmingham, Birmingham, UK \\ ${ }^{5}$ Centre for Computational Biology, University of Birmingham, Birmingham, UK \\ * Correspondence to iain. johnston@uib.no
}

\begin{abstract}
Bioinformatics is a highly interdisciplinary subject, with substantial and growing influence in health, environmental science and society, and is utilised by scientists from many diverse academic backgrounds. Education in bioinformatics therefore necessitates effective development of skills in interdisciplinary collaboration, communication, ethics, and critical analysis of research, in addition to practical and technical skills. Insights from bioinformatics training can additionally inform developing education in the tightly aligned and emerging disciplines of data science and artificial intelligence. Here we describe the design, implementation, and review of a module in a UK MSc-level bioinformatics programme attempting to address these goals for diverse student cohorts. Reflecting the philosophy of the field and programme, the module content was designed either as 'diversity-addressing' - working towards a common foundation of knowledge - or 'diversity-exploiting' - where different student viewpoints and skills were harnessed to facilitate student research projects 'greater than the sum of their parts'. For a universal introduction to technical concepts, we combined a mixed lecture / immediate computational practical approach, facilitated by virtual machines, creating an efficient technical learning environment praised in student feedback for building confidence among cohorts with diverse backgrounds. Interdisciplinary group research projects where diverse students worked on real research questions were supervised in tandem with interactive contact time covering transferable skills in collaboration and communication in diverse teams, research presentation, and ethics. Multifaceted feedback and assessment provided a constructive alignment with real peer-reviewed bioinformatics research. We believe that the inclusion of these transferable, interdisciplinary, and critical concepts in a bioinformatics course can help produce rounded, experienced graduates, ready for the real world and with many future options in science and society. In addition, we hope to provide some ideas and resources to facilitate such inclusion.
\end{abstract}

\section{Introduction}

Bioinformatics is 'one of the fastest-growing interdisciplinary sciences of [...] the early 21st century' [Ai et al., 2012]. The topic is defined in various different ways, but a well-known description is: 'applying informatics techniques (derived from disciplines such as applied maths, computer science, and statistics) to understand and organize the information associated with [biological] molecules' [Luscombe et al., 2001]. A central theme throughout such definitions and descriptions is the interdisciplinary nature of the topic. Bioinformatics projects, like projects in broader data science fields, often involve collaborations between researchers and stakeholders from diverse scientific and societal backgrounds, necessitating training in cross-disciplinary working and a set of communication and co-operation skills in addition to technical competence.

As a modern and highly applied academic field, bioinformatics teaching and practise is also tightly connected with broader trends in biomedical and environmental science and society. The unthinking use of technology in the era of big data, data science, and artificial intelligence can lead to a plethora of scientific, societal, and ethical problems [loannidis, 2005]. In particular, the growing presence of bioinformatics both in health technology and environmental science means that errors in analysis and critical interpretation can have profound consequences 
for human health and environmental policy respectively [Goodman and Cava, 2008, Fulekar, 2009]. New and developing issues associated with the acquisition, storage, and analysis of biological samples and personal data also necessitates an introduction to the ethics of bioinformatics research [Taneri, 2011, Amer, 2017, Goodman and Cava, 2008]. Responsible bioinformatics requires not just a knowledge of tools but a broader critical mindset for continual evaluation of the approaches that one takes [Pevzner and Shamir, 2009, Goodman and Dekhtyar, 2014]. Furthermore, as a comparatively new field, the alignment between taught bioinformatics skills with the changes in perception of, and structure in, the graduate workforce has yet to equilibrate [Hersh, 2008, Ranganathan, 2005, Tomlinson, 2008]. Several regions have explicitly invested broadly in developing bioinformatics skills to address this gap in the research and industrial sectors [Koch and Fuellen, 2008, Tastan Bishop et al., 2014]. Importantly, bioinformatics should not be privilege of the selected few 'bioinformaticians', but be embraced by scientists across the life sciences; thus enabling them to better use their data, or at least understand what data science can do for them. In aligning educational approaches to societal needs, a pervasive question is whether bioinformatics education involves (and should aspire to be) 'educating scientists' or 'training technicians' [Hack and Kendall, 2005b]. Clearly, bioinformatics education can involve a diverse set of challenges, from the spread of desired outcomes and corresponding curriculum contents, through the genuine risks associated with misunderstanding, to the diversity of student cohorts.

Due to the comparative youth of the discipline, pedagogy in the field is young, necessarily technology-coupled and research-focused, and debated [Pevzner and Shamir, 2009, Magana et al., 2014]. As the discipline evolved from disconnected individual approaches to its own field, the formalisation of bioinformatics teaching followed Altman [Altman, 1998] who called for a set of topics for the 'ideal' bioinformatics graduate program. Even this early set of topics suggested pedagogical challenges, including a disparate range of subjects and having to cater for students from a range of backgrounds and with diverse skill sets, from biologists to computer scientists. Since then, the field and its associated pedagogical literature has developed quickly. Curriculum proposals have expanded and diversified, remaining debated [Magana et al., 2014, Koch and Fuellen, 2008, Tastan Bishop et al., 2014, Pevzner and Shamir, 2009]. The 'shrinking half-life of knowledge' [Gonzalez, 2004] is noticeable every day in the discipline; a striking example of this during the design of the course was in a module lead discussion about recommended reading for students, where the difficulty of constructing a reading list was noted, given that specific methodological details in books run the risk of becoming outdated over the timescale of publication. Indeed, Magana et al. propose that these rapid technical advances should be quickly integrated into education and training, to ensure that students emerge 'ready to take jobs and conduct research in these emerging fields' [Magana et al., 2014].

This evolution of the discipline from the ad hoc application of disconnected approaches to its own recognised discipline has been mirrored in the evolution of bioinformatics teaching in higher education institutions. As Furge et al. [Furge et al., 2009] discuss, and Magana et al. [Magana et al., 2014] survey, bioinformatics teaching at an institution typically follows one or both of two paradigms: (i) decentralised bioinformatics content in courses contained primarily within other topics; and (ii) centralised courses with a largely bioinformatics focus. The fast development of the field, and its rapid inclusion into many different biological topics, raises questions about how best to include bioinformatics into existing teaching [Cummings and Temple, 2010] and whether vertical or horizontal integration paradigms should be followed [Furge et al., 2009]. Debate on good practise in bioinformatics teaching continues to spark new initiatives and proposed 'best practises' [Schneider et al., 2011, Welch et al., 2014, Cattley and Arthur, 2007]. Of particular note given the aforementioned issues in bioinformatics applications was a striking absence of ethics content in bioinformatics curricula [Taneri, 2011].

In tandem, bioinformatics as a general discipline (and the related and emerging field of data science) aligns tightly with the connectivist paradigm proposed by Siemens [Siemens, 2005]. Problems in bioinformatics are often best solved through international, technology-assisted connectivism: the use of community messageboard resources; distributed and open-access software; and the global distribution of source data itself. Correspondingly and almost tautologically, computer-based learning is generally deemed essential in bioinformatics education [Cummings and Temple, 2010]. Siemens [Siemens, 2005] highlights a role for 'diverse teams of varying viewpoints [as] a critical structure for completely exploring ideas', aligning with team and active learning and making a strength of diversity in the classroom.

Finally, bioinformatics not only provides excellent opportunities for research-led teaching [Brown, 2016, St Clair and Visick, 2010], but indeed is hard to imagine being taught in any other way [Goodman and Dekhtyar, 2014]. Challenge-based learning, including 'student-centred approaches such as inquiry learning and collaborative learning' was identified as the most common mode of bioinformatics education in a survey of approaches [Magana et al., 2014]. Engagement with open research questions can demonstrate valuable cutting-edge concepts to students as well as serving to motivate and justify the reason for learning about these topics and exposing them to a realistic representation of the field beyond education. 
These ideas can be synthesised and distilled to identify a set of positive principles for bioinformatics education (and broader data science education), although the weighting of these principles varies across studies. These general positive principles emphasise problem-based learning and self-learning [St Clair and Visick, 2010, Ranganathan, 2005] and often adopt a jointly cognitivist and constructivist strategy with (i) an intensive introduction to the range of necessary concepts and (ii) subsequent focus on a subset of skills motivated by a specific problem [Goodman and Dekhtyar, 2014, St Clair and Visick, 2010], following a constructive alignment approach [Biggs, 1996]. The goal of the project outlined in this report was to realise this paradigm while supporting student development of ethics, critical analysis, and transferable skills, thereby furnishing graduates with technical and transferable skills that increase their diversity of options in the workplace and in society. We hope here to provide insights, references, and resources that may be of value in the design of other bioinformatics and broader courses aiming for this goal in the future.

\section{Interdisciplinary and transferable skills in a bioinformatics course: cur- riculum design and review}

The module described in this article is titled 'Interdisciplinary Group Project in Bioinformatics', and is a 20-credit part of a recently-designed UK MSc Bioinformatics course (UK FHEQ Level 7). This course takes graduates from a range of topics (computer science through biology), with a 2:1 entry requirement. Cohort size so far have been in the range of 10-30 students. The other modules are Essentials of Mathematics and Statistics (20 credits); Genomics \& Next Generation Sequencing (20 credits); Data Analytics \& Statistical Machine Learning (20 credits); Metabolomics and advanced (omics) technologies (20 credits); Computational Biology for Complex Systems (20 credits); and an Individual Research Project (60 credits).

The interdisciplinary module was designed around the paradigm of constructive alignment [Biggs, 1996], with the essential aspects of the above section which were desired to be captured:

- Progression through a taxonomy of learning [Krathwohl, 2002], from knowledge of tools to their application and critical evaluation;

- Introduction to a research environment;

- Group work facilitating peer-assisted, active learning [McKinney, 2010] and necessitating the development of co-operation and communication across a range of backgrounds [Pevzner and Shamir, 2009];

- Development of skills that are valuable outside the specific academic field of bioinformatics.

We considered the delivery of a genuine bioinformatic research project by an interdisciplinary group of students as the ideal 'desired result' that would provide evidence of these targets [Wiggins et al., 2005, Magana et al., 2014]. To this end, a format was decided where students work in groups of 2-4 to address an open-ended research question under the supervision of an academic at the institution. This reflects two principles for the promotion of learning from Merrill [Merrill, 2002] highlighted for bioinformatics by Cummings \& Temple [Cummings and Temple, 2010]: learners are 'engaged in solving real-world problems' and and 'new knowledge is applied by the learners'. Taught, discussion, and practical exercise content is presented in tandem, involving introductory technical skills and transferable concepts including how to prepare scientific publications, posters and presentations as well as on the ethics requirements of research. The formal module outcomes are given in Table 1 and linked to the general QAA principles for an FHEQ level 7 course and for biosciences qualifications in Appendix A [Hack and Kendall, 2005a].

In the design of this module, the ideas from the introduction above have naturally crystallised into a multifaceted strategy. There is a role for a limited cognitivist paradigm in those situations where a correct answer does exist (for example, choosing an appropriate statistical test or a particular piece of software) which can be used to build a consistent knowledge platform across a diverse class. We can then naturally build upon this knowledge foundation to progress chronologically through Bloom's taxonomy of learning [Bloom et al., 1956, Krathwohl, 2002]. Through introductory technical lectures, students acquire knowledge (specific tools, statistical tests and so on, building a consistent platform across the diverse student backgrounds in the class), and comprehension (how these specific processes relate to each other and to scientific questions). Motivated by an open research question [Cummings and Temple, 2010, Brown, 2016], and working in diverse teams [Siemens, 2005], the students will develop, apply, synthesise, and critique their own bioinformatics approaches [Krathwohl, 2002] through a jointly social constructivist and connectivist strategy [Kim, 2001, Siemens, 2005]. They apply these technical concepts in parallel with the interdisciplinary skills in collaboration, communication, and ethics that are developed through the module as they design, implement, critique, and present a real group research project [St Clair and Visick, 2010]. The social constructivism inherent in the group project structure [Kim, 2001] is harmonious with the targets 
By the end of the module students should be able to:

(a) Work effectively in an interdisciplinary team;

(b) Carry out a relevant literature search for their topic;

(c) Demonstrate a comprehensive understanding of the broad world of omics in the context of complex biological, clinical, or environmental data;

(d) Choose appropriate computational and/or mathematical approaches to perform analysis of omics data; evaluate methodologies and develop critiques of them and, where appropriate, propose new methods;

(e) Demonstrate a systematic understanding and critical awareness of the implications of research in biology-related fields, including an understanding of ethics;

(f) Present the results of the project in written and oral form.

Table 1: Module outcomes.

of developing communication and teamwork skills, and with learning via 'belonging to something' [Hodkinson et al., 2004] via shared problem-solving [Collin and Valleala, 2005].

The students must demonstrate extensive and critical knowledge of ideas in the field in order to design their strategy to address the research question, which is, by construction, an original application of this knowledge. In addition, they must creatively deal with typical issues that aries from a real research project such as absence of anticipated data, computational-logistical issues and teams shrinking as students left the course.

The module is geared towards goals of developing self-direction and acquisition of transferable skills including ethics and communication, supported by peers and supervisors while developing an individual trajectory. The module is reviewed every year with module leads, student representatives, and an external examiner. In harmony with positive feedback from students and the external examiner, the outcomes of this review so far have been to keep the learning outcomes the same while redesigning some timetabling aspects to give students more time to independently absorb ideas (Appendix B.II). Future monitoring of the evolving literature in the field and utilisation of instructor and peer experience on the role of the subject in society allows ongoing reflection on this design.

\section{Interdisciplinary and transferable skills in a bioinformatics course: mod- ule content}

A consistent challenge in bioinformatics teaching is the diversity of student cohorts and the corresponding technical backgrounds. This module aimed to address this challenge with a dual approach. First, a 'diversity-addressing', non-examinable set of foundation lectures and exercises ensuring that important core concepts from both biology and computing, as well as important transferable and interdisciplinary skills, were met and engaged with by all students. Second, a 'diversity-exploiting' strategy where the strengths of students' diverse backgrounds were brought together to be greater than the sum of their parts.

\section{Introductory philosophy: information and life}

The first 'diversity-addressing' foundational content required non-biologists to meet core biological concepts and biologists to meet key informational concepts. This was addressed by first providing a set of basic biology lectures with an 'information and life' flavour, and next providing an entry-level set of coupled lectures and exercises on core technologies.

The introductory lectures started with the pitch that 'all biology is computational biology' [Markowetz, 2017] that is, modern biology requires computational methods, and 'computational biology' will soon become as redundant as 'pipette biology'. Some classical high-profile bioinformatic studies, with simple core concepts and strikingly visual outputs, were summarised (for example Novembre et al.'s finding that European genetic structure closely aligns with physical geography [Novembre et al., 2008]). Aligning with this focus on computation and information, life was presented as the reproduction and maintenance of information in the face of entropic disorder. The central dogma of molecular biology was covered from an informational perspective - following the transfer of information between different molecules to facilitate function in the cell, through information storage and use (the central dogma), replication (evolution), and processing (signalling and regulation). Pursuing 'life as information' further, the Kolmogorov complexity associated with life's information content [Edmonds, 1995] was heuristically 
illustrated with an exercise using gzip to compress photographs of a rich jungle ecosystem (1.4MB compressed to $1.2 \mathrm{MB}$ ) versus a dead moonscape (1.4MB compressed to $644 \mathrm{~KB})$; and the human genome (779MB compressed to $676 \mathrm{MB}$ ) to explicitly link 'biological' with the more familiar 'computational' information. The idea that Kolmogorov complexity can describe evolved 'algorithms' that give rise to apparently more complex outputs was probed further using L-studio to simulate plant growth and form [Prusinkiewicz et al., 1999].

A group exercise followed where students from biological and non-biological backgrounds were paired, asked to choose one of a list of biological topics, and after a short period for preparation and research, give a minute's presentation on informational aspects of their chosen concept. This was expanded into an overview of some examples of topics of bioinformatic study (transcriptomics, proteomics, metabolomics, etc) and the information storage, replication, and/or processing involved in each case. Finally, an aside on artificial life [Levy, 1993], Von Neumann machines [Von Neumann et al., 1966], cellular automata [Conway, 1970], and the Tierra / Avida environment [Ray, 1991, Ofria and Wilke, 2004, Adami et al., 2000] was presented as an informal and visually appealing example of the relationship between information and (biological or artificial) life.

\section{Teaching computational core concepts with virtual machines}

The first technical aspect of this module is an initial intensive introduction to various central software approaches in bioinformatics. Based on previous experience and positive feedback from students, and following the paradigm of active learning [McKinney, 2010], we employed intrinsically mixed lecture delivery and computational practical classes. Here, students are introduced to a set of computational ideas (for example, the structure of a file system, or a particular set of commands) and then immediately perform a task involving those ideas on their laptops. The idea is to couple the theory with an immediate practical application, thus reinforcing the concept in student's minds [Yazedjian and Kolkhorst, 2007] and giving them first-hand constructive experience of a genuine application. Here, both an instructor and peers are on hand to help with troubleshooting, breaking down barriers of anonymity [Michaelsen et al., 2002] and facilitating peer-assisted learning [Johnston, 1997].

An issue in previous experiences with this mode of working was inconsistency: what worked on one student's computer might not work on another; some may use Macs and others Windows machines; and so on. As Dahlö et al. [Dahlö et al., 2015] point out, 'if a student tries to run a command from the instructions and it results in an error message, it can be hard for a beginner to figure out what went wrong... every problem they encounter with the operating system steals focus from the subject being taught'. This is perhaps not the only issue; it can be highly demoralising for students who, faced with an unfamiliar interface or application, fail to get something working for technical reasons. This issue is amplified by the diverse student backgrounds in a bioinformatics class with a range of computational abilities and experience. This was anecdotally illustrated to one of us (IGJ) in a separate, undergraduate computational biology practical session: students faced with between-computer inconsistency were both frustrated and disillusioned about the 'rigour' of the topic (and of the instructors).

To pre-empt this issue, we use a 'virtual machine' (VM) environment. VMs are simulations of a complete computer system that can be constructed exactly as the instructors require and are accessed remotely by the students using their laptops (Fig. 1). We therefore ensure that each student sees the exact same computational platform, and prior to the class ensure that all commands and approaches involved will function as expected. The instructor's own behaviour on the same VM environment is projected to the class.

The specific VM environment was part of a wider infrastructure in total comprising 48 nodes with between 20 and 40 cores and 128+GB RAM each. The VMs presented to the students had 2 virtual CPUs and 14GB RAM. Secure Shell (SSH) connections (via Putty on Windows or the terminal on Mac/Linux machines) with graphics forwarding enabled was used to connect to these VMs, which presented students with a Bash command line interface through which Bash commands, Python code, R code, and other software could be run. Some students used this environment for their later research projects, which entailed increasing the amount of computational resource associated with those VM instances. The technical topics covered here included an introduction to working with the command line, Python, R, and Git.

Command line. The command line section involved a joint student-instructor exploration of Unix file structure (students and instructor used the command line to navigate and explore together a preconstructed example file system) and the Unix philosophy, explored together with the instructor by using grep, sed, awk, and other tools to construct pipelines to analyse preconstructed data. Short single-line exercises accompanied lecture slides on the theory of these commands ${ }^{1}$ Following the joint lecture-exercises delivery, more involved practical exercises

\footnotetext{
${ }^{1}$ Outside of scheduled contact time, students were encouraged to play 'Regex Golf' (http://regex.alf .nu/) to gamify an introduction to this rather technical concept.
} 


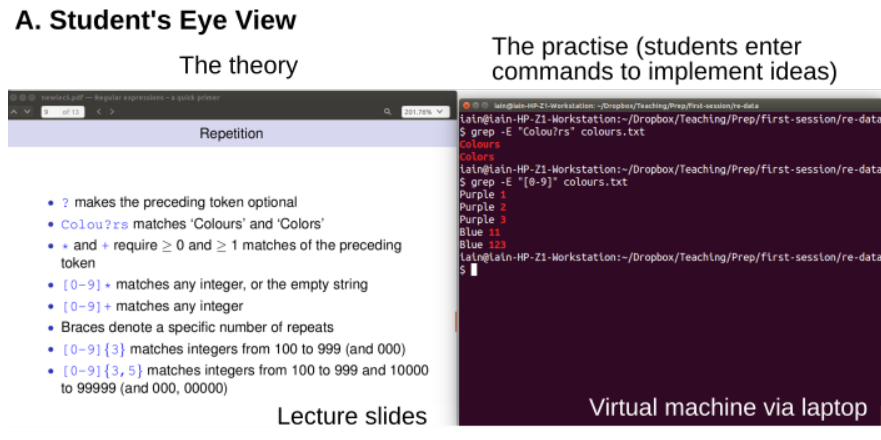

B. Virtual teaching space

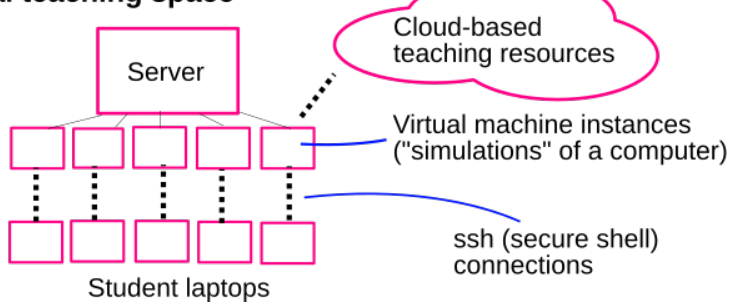

C. Physical teaching space

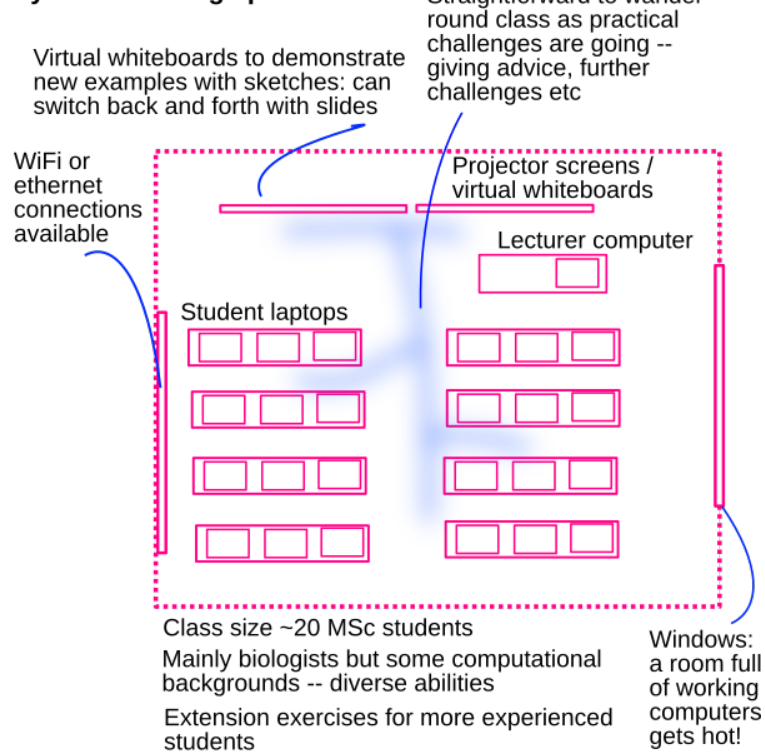

Figure 1: Physical and computational structure of the virtual machine learning space.

were explored, including working in small groups (2-4) to build analysis pipelines for specific bioinformatics problems motivated by the instructor's research (for example, identification of G-quadruplex-forming regions in DNA sequences [Kikin et al., 2006]). The connection with ongoing and current research was emphasised to illustrate the cutting-edge and practical nature of these modes of working [Brown, 2016, St Clair and Visick, 2010]. The instructor worked in parallel with the class via the VM environment, solving the multistage problem step-by-step with suggestions from the class groups.

Python. In order to teach Python to the students, it was decided to focus on giving them a good grounding in the fundamentals of general programming, then moving on to common concepts before introducing more complex applications. We considered alternatives, including concentrating on 'teaching by example' by providing complete code blocks that would cover the main use cases the students would face. However, we believed that this approach could lead to students developing a narrower ability, not only to develop their own code but also to understand and modify third party code. Given that bioinformatics often uses several languages and development paradigms, and indeed that later course content also develops skills and practise in R, we also considered an introduction to general programming techniques as an appropriately transferable introduction.

The Python course was taught in a single intensive block over one and a half days and was principally derived from a $\mathrm{C}_{++}$course taught by one of us (MS) to postgraduate physics students. It started by covering fundamentals such as variables, objects, and operators, before moving on to other general programming concepts such as loops, conditionals and functions. There was a through-line for the majority of the course of writing an implementation of the Caesar Cipher which allowed progressively more involved exercises to be set. The aim of this structure was to give the students as much hands-on programming time as possible during the course and thus develop their code-based problem solving skills, without yet requiring the additional time overhead of solving harder, 'real-world' problems. This approach also fitted well with the text and file manipulation they were most likely to use Python for, at least in the short term. Consistent with the overarching active learning and mixed lecture-practical philosophy, an effort was made to not lecture for more than 20-30mins before providing another hands-on exercise. In addition, where appropriate, both the Python interpreter and scripting were used in order to give the students practise in both.

Overall, student reception to the course was positive (Appendix B), and even the students that started off the course with no programming knowledge had grasped the basic concepts by the end. Their uptake of basic programming concepts was demonstrated by their familiarity with loops, conditionals, data structures and so on in later $\mathrm{R}$ practise, underlining the 'diversity-addressing' results of this approach. The basic course structure was retained through the years it has been run; however, we have recently decided to move away from the Caesar Cipher example and employ a more bioinformatics-related 'gene manipulation' example. This task will have a similar level of difficulty but provide a more direct connection to biological data.

This is one part of the wider module where more time could be readily invested in more detailed and/or thorough 
coverage of core concepts. Going forward, it was thought that outcomes would be even better by spreading this content out over a longer period, thus allowing students time for reflection on the material, and the setting of 'homework' to which feedback could be given. These ideas were also raised in student feedback (Appendix B). However, as a necessary precursor to other content in the course, there is a logistic tension between time spent here and time on other more specific content later. Given the restrictions of the broader course timetable, we currently retain this short, intensive introduction format, with a view to more advanced and specific concepts being covered in subsequent modules as required.

R. The concepts met in the Python introduction were next translated into an introduction to the $R$ statistical environment [R Core Team, 2015]. Given their importance in bioinformatics, particular attention was focused on the variety of data types available in R. Complementing the previous Python coverage, exercises in program control and input/output were completed in groups of 2-4 as before. A brief introduction to implementing simple hypothesis testing in $\mathrm{R}$ was demonstrated, but more statistical details were withheld for the future module on that topic. Finally, an interactive visualisation practical was held, first using R core capacity and then the ggplot2 library [Wickham, 2009]. Students were very engaged in these visualisation practicals, enjoying exploring the styling options afforded by $\mathrm{R}$ and particularly ggplot2, and working in an open-ended manner to compete and co-operate in producing beautiful visualisations of data.

Git. Due to time constraints, an introduction to version control and Git was presented in a light-touch format. Again working jointly with the instructor on the command line, students forked an existing repository from GitHub (www.github.com), proposed changes and submitted pull requests, which were accepted by the instructor in real time to demonstrate both sides of the process.

Experience with the joint student-instructor exploration of topics with the VM teaching environment was very positive. The infrastructure needed to access VMs took some time to install, but the students were informed of this before the session, more staff were present to help, and a light-hearted environment prevailed through this short technical phase. Subsequently, content delivery was smoother and completely consistent between teacher and student (and between students), increasing accessibility across the diverse student cohort. Students readily helped each other out with specific commands in the uniform environment; individual and group exercises progressed very smoothly. The environment was explicitly praised in student feedback, with positive reception of 'activities in the [programming] sessions to reinforce our learning' as 'a good way to understand what I have learnt'. Particularly encouraging was praise for the approach which 'really helped build confidence' and 'meant students from a non-computing background could follow along without feeling too lost' (Appendix B.I).

Interestingly, while VMs are used in computer science education [Lathan et al., 2002], there seems to be limited literature examining their use in bioinformatics teaching [Afgan et al., 2015, Dahlö et al., 2015]. The VM environment has several potentially powerful strengths and extensions, one of which is its natural provision of data for learning analytics. Commands that students enter in the VM are stored and can be retrieved: these data can be used to explore, for example, which commands students experience the most trouble with, and the amount of time students spend on different exercises, so that attention can be focused on more problematic areas.

\section{Interdisciplinary and transferable skills in bioinformatics}

The learning outcomes in Table 1 require several skills that are naturally 'transferable' in nature (although take some specific forms in the field of bioinformatics). These include (i) the ability to work effectively in an interdisciplinary team; (ii) to present the results of the project in written and oral form; and (iii) to demonstrate critical awareness of the implications of research in biology-related fields, including an understanding of ethics.

To address these goals, we held an ongoing series of seminars and exercises on the topics of: (i) structuring a scientific investigation, logistics of bioinformatic investigation, and communication in interdisciplinary groups; (ii) scientific writing and delivering research; and (iii) scientific methods and philosophies, bioethics, and ethics in bioinformatics. These topics (detailed below) were presented in a series of two-hour joint lectures and exercise classes, as with the technical content delivered previously.

\section{Structuring and logistics of investigation, and scientific communication}

The introduction to structuring an investigation was intended to give students a grounding in some recommended steps (and a possible ordering of those steps) for a successful research project, prior to their embarking on the group project itself. These steps were presented as 'phases': specifically, introductory, exploratory, development, science, analysis, delivery. Flexibility in this ordering - including loops - was explicitly supported. We particularly focused on introductory (literature searching, discussion, crystallisation of research questions) and exploratory 


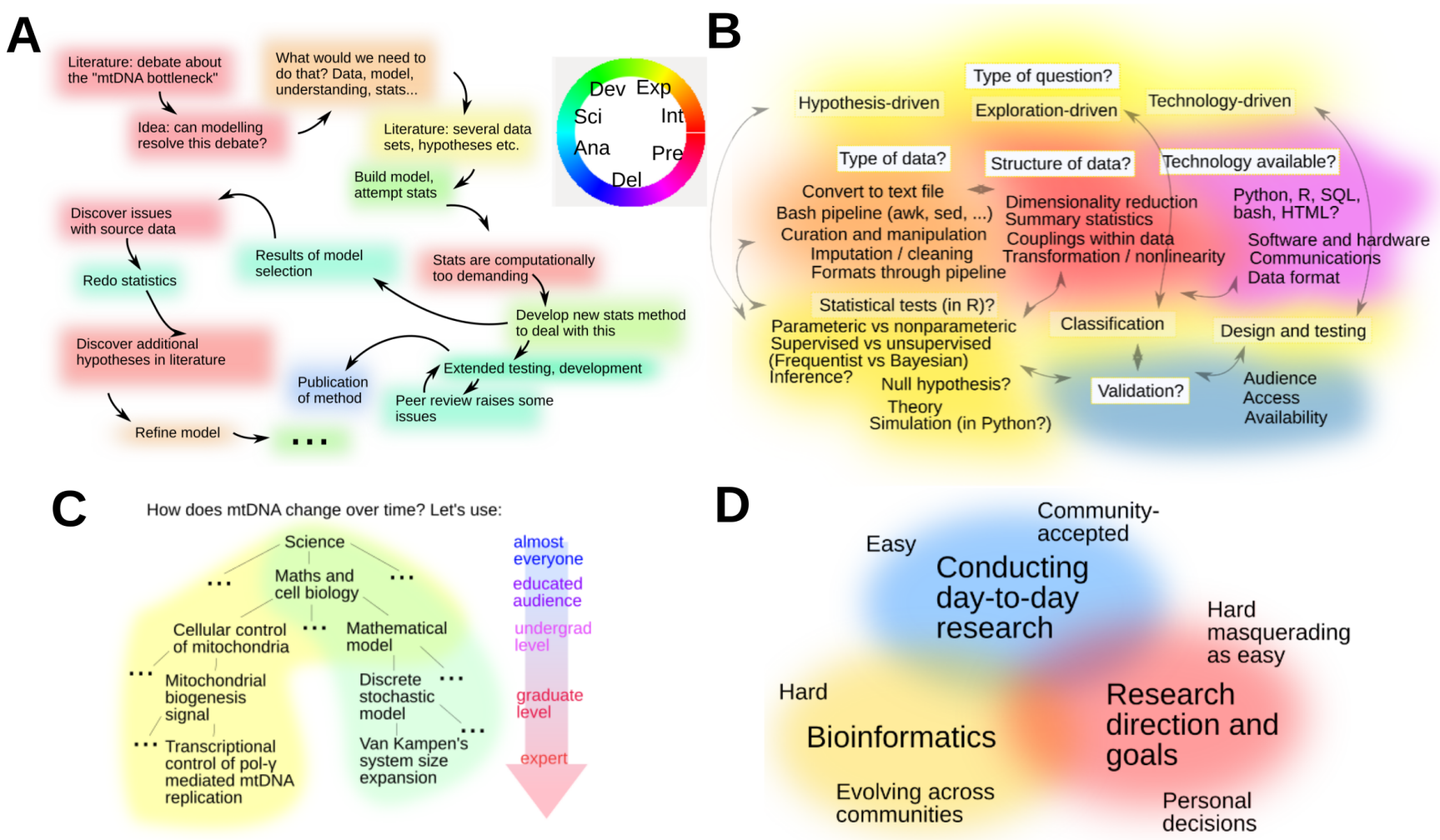

Figure 2: Illustrative concepts in interdisciplinary and transferable topics. (A) 'Decision bush' for logistics of a bioinformatic investigation, illustrating some different types of research projects, and the (coarse-grained, and sometimes overlapping) general approaches that can be used in different cases. (B) The evolution of a real research project, showing branching and backstepping. Colours broadly refer to Introductory, Exploratory, Development, Scientific, Analysis, Deliverable, and Presentation phases. (C) A 'phylogeny of jargon' for interdisciplinary communication in bioinformatics groups, with the 'comfort zones' of two different researchers and their intersection for a specific subset of topics. (D) A fuzzy Venn diagram of ethical perspectives between general 'dayto-day' science (where principles of core good practise are generally accepted), specific bioinformatics practice (where principles of good practise are evolving and new issues are arising), and individual researcher priorities (where personal ethics require consideration).

(here, data curation and exploration) phases as important for new researchers to take time over. A real project from one of us (IGJ) was presented to the students to illustrate the many backs-and-forth between these phases that research can take, and to reinforce that backtracking, abandoning research paths, and diverging from the original plan are natural dynamics in research (subset shown in Fig. 2A). General principles - realistic milestones, codifying and updating goals, keeping colleagues and PIs up to date - were also highlighted.

In the subsequent 'logistics' discussion, we focused on 'what is the question?', 'what data is available?', and 'what tools and technologies can we use?'. In particular we highlighted that a bioinformatics question can be scientific ('does gene expression change in disease?'), exploratory ('what happens after exercise?'), or technological ('can we build a tool to interrogate and visualise this dataset?'). Students were encouraged to contribute thoughts on choosing bioinformatics pipelines as well as statistical and visualisation approaches appropriate for the combination of question and data (hypothesis-driven vs exploratory, categorical/continuous/high-dimensional data, and so on). In particular, the philosophical difference between hypothesis testing and exploratory, hypothesis-free research (as is often the case in bioinformatics, where data-driven exploration is used as a process for hypothesis generation) was highlighted. A 'decision bush' (Fig. 2B) was suggested as an amalgamation of these overlapping routes. We also focused on the message that results should be robust with respect to choices made in the analysis pipeline (e.g. particular parameters, thresholds, etc should give the same qualitative results).

Bridging these topics in research design and the students' actual group projects, we next discussed communi- 
cation in and to interdisciplinary groups. Students were encouraged through lectures and exercises to consider a 'phylogeny' of technical language, starting with very basic 'ancestral' terms like 'life' and 'science' and progressing, as a participant's specialisation increases, to more technical terms 'gene', 'stochastic model', and so on (Fig. 2C). Students were encouraged to identify the 'common ancestor' terms for a particular topic with a given imaginary conversational partner (a schoolchild, a physicist, an experimental collaborator) and build terminology from there. We also discussed 'unspoken' conventions in different disciplines that may be graphical (for example, the box plots and significance stars that are ubiquitous in biology but rare in physics), verbal (different abbreviations), quantitative (different interpretations of statistical significance, $p<0.05$ in biology vs roughly $p<3 \times 10^{-7}$ in physics - the $5 \sigma$ threshold for 'discovery'). Differences in scientific philosophy (heavily hypothesis-focused in biology, exploratory model-based in physics, data-driven exploration in bioinformatics) and career priorities (publication strategy and emphasis of bibliometrics including impact factor, authorship conventions, and expected grant incomes were also mentioned as potential sources of tension in interdisciplinary groups. These discussions drew on the instructor's experience of working in different academic fields and different departments (specifically physics, maths, and biology departments).

Finally, some informal discussion of Belbin's team roles [Belbin, 2012], academic personalities, and good practise in small team dynamics [Salazar et al., 2012] was held. In particular, a study summarising the meeting dynamics of innovative teams was explored [Salazar, 2018]. Here, team reflection (at the start and end of a meeting), promotive voice (at the start of a discussion), perspective seeking (as ideas develop) and managing interdisciplinary connections were the recipe for innovative and productive meeting. The idea here was not to insist upon employing ideas from management studies, but to make students aware of some existing ideas about beneficial combinations of skills, approaches, and collaboration dynamics. The plasticity of team roles - that a given researcher may be called upon (or find it desirable) to change roles throughout a project - was highlighted in conjunction with the expected plasticity of interdisciplinary scientific research projects above.

To develop skills in research communication, the students took part in an interactive scientific writing exercise hosted by institutional experts, with followup class discussion on the basics of scientific publication, posters, and talks. Students were asked to name journals that they had come across; this growing list was augmented with some of the instructor's suggestions in both interdisciplinary science and the bioinformatics field. Bibliometrics, audiences, publication costs, and other issues in choosing a journal were discussed. Mensh and Kording's 'Ten Simple Rules for Structuring Papers' in PLoS Computational Biology [Mensh and Kording, 2017] were introduced, leading to ideas for structuring striking research posters, and general principles for giving a good scientific talk following Alon's brief but highly targeted paper [Alon, 2009]. Issues in scientific publication, including preprints, open access, double-blind review, and gender bias [Borsuk et al., 2009] were also briefly presented.

\section{Scientific methods and philosophies, and ethics in bioinformatics}

Linking with the previous mentions of different philosophies of science in different disciplines, a brief overview of general scientific philosophy was discussed with students. This included a historical perspective illustrated through evolving theories of astronomy, a discussion of deductive, inductive, and abductive reasoning, and Kuhn's structure of scientific revolutions [Kuhn, 2012]. The discussion then turned to why these ideas are important for bioinformatics. As a comparatively new field, bioinformatics can be viewed as a (gentle) revolution, where new paradigms of interdisciplinarity, computational focus, and hypothesis-free investigation are reshaping traditional modes of biological investigation [Hack and Kendall, 2005a]. The risk of overemphasis on buzzwords and newer, less interpretable ways of working (as with any exciting new discipline) was discussed, with some focus on 'mistakes from early Genome-Wide Association Studies (GWAS)' [Lambert and Black, 2012] and highlighting the need for critical analysis of pipelines and published results. It was striking how many students considered the appearance of work in a peer-reviewed journal as a signature of 'gospel truth'; the importance of critically assessing published methodologies and interpretations was emphasised through several case studies. These included the dramatic observed differences between lab animals and their wild relatives [Abolins et al., 2017]; the fact that mice respond differently to male and female human researchers [Sorge et al., 2014]; the well-known critique of statistical approaches in biomedical sciences suggesting that 'most published research is false' [loannidis, 2005]; and the reproducibility crisis across scientific disciplines [Collaboration et al., 2015] including drug discovery for cancer, where only $11 \%$ of pre-clinical research results were found to be reproducible [Begley and Ellis, 2012], and Phase II success rates had fallen to $18 \%$ in 2011 [Prinz et al., 2011].

This coverage of issues in scientific publishing and practise led naturally to a discussion of bioethics. The students were asked to construct a 'code of practise' - a set of tenets that they considered to describe ethically sound research. These points were then compared with the instructor's set of ideas. Frequently there was substantial 
overlap, with students demonstrating an awareness of plagiarism, forgery, and transparency. Topics highlighted by the instructor that were less commonly noted by students included personal aggression and discrimination in research interactions, distinguishing evidence from speculation, and appropriate assignment of credit via citations or other referencing.

This comparison of ideas between students and instructor set the tone for the future coverage of ethics topics. Students and instructor took part in guided but free discussion of several ethical topics in research, (from core scientific practice to bioinformatic-specific issues) through the lens of the individual researcher (Fig. 2D). These first included the role research plays in society as a whole, from the broad ('what should science achieve?', 'who should pay for it?') to the specific ('how can we justify the carbon costs associated with large-scale computational biology?' and the ' $10 / 90$ ' gap, where only $10 \%$ of health research funding is spent addressing $90 \%$ of the world's health problems [Schüklenk and Kleinsmidt, 2006]).

Following the previous examples of scientific philosophy, we briefly touched upon different ideas of 'doing good', from the principles of biomedical ethics [Beauchamp et al., 2001] to Bentham's 'greatest happiness of the greatest good', and Pearce's associated 'hedonistic imperative' of the abolition of suffering through the use of biotechnology [Pearce, 1995]. Considerations for individual researchers choosing a research project or direction were discussed, including the individual's personal motivations, the likelihood that the 'advertised' benefits of the research will be achieved, and the balance of contributions and costs associated with the project. Students were encouraged to consider which scientific disciplines and individual scientists had saved the most lives in history; subsequently we explored the thought-provoking website 'Science Heroes' (http://www.scienceheroes.com) which attempts to estimate this latter quantity. That website suggests Fritz Haber, responsible for the nitrogen-fixing Haber process and hence the development of synthetic fertiliser, as the top of this list. Haber provides an interesting discussion point on this topic. On one hand, his Nobel-prize winning invention has saved (or allowed) estimated billions of human lives. On the other, he was one of the fathers of modern chemical warfare, directing and supervising the use of chlorine gas on the battlefields of World War I. These dramatically different contributions from the same man led to class conversation on the inherent 'goodness' of particular research topics, the moral compass behind research intentions, and decoupling research from researcher.

We next discussed whether the issues that often motivate bioinformatic studies are best addressed through fundamental science, public health, or other policy interventions. Several examples were used to crystallise this discussion, including the topic of plant science and food security. Substantial scientific and bioinformatic effort is spent characterising, at the molecular level, crop plant traits that may improve productivity and improve food security. However, a third of the world's food is simply lost or wasted every year [fao, 2019], eclipsing the gains from many molecular insights. Students were encouraged to discuss whether societal resource was best spent addressing these logistic issues, more scientifically advanced biotechnology, or both. We found that most students had not previously connected a scientific project to a real economic cost to society (for example, grant funding from a research council). When presented with costs of some real research grants, and the overall budget of UK research councils, they were able to compare research and policy solutions on a more even footing.

Focusing increasingly on bioinformatics, students were asked to populate a list of what they considered ethical topics particularly pertinent to the field of bioinformatics. Students initially had difficulty in compiling this list, perhaps suggesting that they had rarely considered ethical concerns in the field previously. 'Designer babies' was often among the first ideas to appear [Amer, 2017], and 'personal data' appeared in several suggestions. Correspondingly, the next point of discussion was from the Exome Aggregation Consortium (ExAC) study, highlighting the possible overselling of 'pathological' variants discovered in early bioinformatics investigation [Lek et al., 2016]. This study revealed that subjects had, on average, 54 mutants that were considered pathogenic, but about 41 of these were so prevalent in the human population that their pathogenicity is debatable. The fact that these misclassifications affected real people's lives led to a call for increased scrutiny of functional mechanisms before pathogenic variants are declared from bioinformatic analysis alone [Editorial, 2016].

We next focused on the structure, acquisition, and use of bioinformatic data. We discussed a 2016 study highlighting the traditional ethnic focus of GWAS on participants of white European ancestry: $81 \%$ of samples (down from 96\% in 2009) across studies were of European ancestry, and 14\% of Asian ancestry [Popejoy and Fullerton, 2016]. This was striking news for our students, working in classes of rather international background, and set the stage for a discussion of how global the benefits of GWAS are, and should aim to be [Bustamante et al., 2011]. Following this point, the heterogeneous worldwide impact and benefits of bioinformatics research was next discussed, with particular reference to 'benefit sharing', defined by Ref. [Schroeder, 2007] as '...the action of giving a portion of advantages/profits derived from the use of human genetic resources to the resource providers in order to achieve justice in exchange.... A critical ethical and legal analysis of north-south benefit sharing in genetics research [Schüklenk and Kleinsmidt, 2006] was discussed, along with a critique debating whether and 
how developing countries will benefit from their participation in genetics research [Ndebele and Musesengwa, 2008].

We next covered a current issue in the ethics of bioinformatics - the re-identifiability and the subsequent potential for misuse of personal data [Homer et al., 2008, Greenbaum et al., 2011]. Students had generally met ideas of anonymising and pooling genetic data, but were surprised at the ease with which individuals could be re-identified under many of these circumstances [Homer et al., 2008]. A provocative article on this topic about 23AndMe's use of genomic data [Seife, 2013] was read in depth. This article raised the points of the company's permissive terms and conditions allowing the use of personal genomic information for marketing, and the fact that any family member using such a service is automatically providing a large amount of the genetic information they share with other family members to that service.

Previous studies have highlighted a surprising absence of ethical topics in bioinformatics courses [Taneri, 2011], and given the comparative youth of the field overall, and the ongoing debate about curriculum content, it is perhaps not surprising that a consensus set of central topics has yet to be decided [Amer, 2017]. We propose the above topics - general bioethics; the role of research in society; the risk of biological misclassifications; the global sampling and benefits of bioinformatic research; and the use of personal data, not as a fixed set of recommended discussion points but as an illustrative sub-curriculum to get students thinking, discussing, and critiquing some relevant and important issues in the developing field.

\section{Interdisciplinary group projects}

During the summer prior to the course start in September, academic staff in the natural, sports, and medical sciences divisions were contacted with a soliciting email offering the opportunity to propose bioinformatics projects. The requested response was a detailed abstract for a project outlining the research question and data to be used, as well as some key references for interested students. The suggested format was weekly supervisory meeting with the project's principal investigator (PI) from October to March. Projects were vetted by the module lead for bioinformatics alignment (though no projects to date have been proposed that were unsuitable) and compiled into a booklet. Students were presented with this booklet and asked to rank their top five choices by order of preference. Students were then allocated projects based on their disciplinary and geographical background, their propensity for active participation in class discussion and Q\&A, and their preferred rankings (see below).

The goal in student assignment was to expose students to a diversity of backgrounds and personalities within a group. Diversity of backgrounds was typically easy to achieve; groupings mixed biologists and non-biologists where possible, and biologists of different sub-disciplines where not. UK/non-UK and European/non-European undergraduate locations were also mixed.

Grouping personalities was more challenging. The instructor at this stage in the course had only had limited (though intensive) exposure to the students. At such early stages of the programme we deemed it desirable to have 'some but not too much' difference between the members of a group. Some diversity had many advantages: requiring the students to put into practise the module content on working in interdisciplinary groups; reflecting an accurate picture of a real bioinformatics collaboration [Biggs, 1996, Cummings and Temple, 2010, Wiggins et al., 2005]; working with different priorities, personalities, and team roles [Salazar et al., 2012, Belbin, 2012]; and requiring compromise between different parties' interests and goals. However, we strove to avoid situations where, for example, one domineering personality was paired with several less bombastic partners. While such situations certainly exist in the broader world, we believed that this would prevent all students getting the broadest set of experiences from the group project.

Following existing approaches to explore and develop bioinformatics skills with projects [St Clair and Visick, 2010], the idea here was to expose students to open-ended research questions that were not pre-choreographed, with the associated requirements of project development, management, critical analysis, and overcoming unforeseen hurdles. Projects run in this module included: learning pathways of disease progression for precision medicine; predicting responses to endurance-exercise training; control and function of beta cells; constructing an 'atlas of the phosphatasome'; using ecological and evolutionary dynamics to forecast responses to global change; detour of proteins in cancer; 'metabolism across space and time' and its interpretation; horizontal gene transfer in bacteria; and 'moving towards predictive evolution'. These ranged from more classical bioinformatics (RNA-seq data, enrichment analysis, gene ontologies, pathways) to more unusual goals such as constructing a web resource for a class of proteins (the phosphatasome atlas above). This latter project was particularly interesting: the small group consisted of one student from a computer science background and one from a biology background, leading to mutual reinforcement and cross-fertilisation of ideas, and leading to an output deliverable (a fully functional database and rich graphical web interface populated with biological instances) rather greater than the sum of its 
parts. This project also drew particularly positive peer responses, with other students explicitly noting the benefits of the joint expertise of the researchers.

These projects took place while students were also attending taught content on different topics (modules above). This led to time management issues for some groups, particularly at times where these other topics were being assessed. Two projects involved, to different degrees, an anticipated issue: heterogeneity in workload (or perceived workload) within the group. Some students raised concerns that their colleagues were not sufficiently contributing to the project. The concern was less often around how much research could be achieved and more often about how group marking would reward these colleagues. As discussed below, ongoing review of the assessment structure for this module will seek to address this.

\section{Interdisciplinary and transferable skills in a bioinformatics course: as- sessment design and review}

Several targets were borne in mind when designing and reviewing the assessment structure and its alignment with our goals and learning outcomes. Vitally, and given that 'assessment is the curriculum as far as students are concerned' [Ramsden, 2003], we wished to 'support worthwhile learning' [Gibbs and Simpson, 2005]. The learning outcomes for this module (Table 1), as discussed above, emphasise teamwork, communication, and transferable skills in addition to the technical completion of a research project. Based on our perceptions of the wider role of bioinformatics and its associated skill set in society, we wanted this worthwhile learning to reflect what graduates may require in the workplace as well as in academic settings. We wished to ensure that teamwork was rewarded while also allowing individual flair, and to adequately reward different strengths in a diverse cohort. This approach is inspired by the constructive alignment paradigm [Biggs, 1996] of 'testing bioinformatics by doing bioinformatics' - and 'doing bioinformatics' necessarily involves a combination of transferable and technical skills [Pevzner and Shamir, 2009, Goodman and Dekhtyar, 2014].

Two particularly pertinent points from Gibbs \& Simpson [Gibbs and Simpson, 2005] naturally crystallise a reflection on assessment: (i) that 'tackling the assessed task engages students in productive learning activity of an appropriate kind' and (ii) providing timely, objective, appropriate, actionable feedback.

For (i), we designed assessment as a multifaceted test of technical and transferable skills. Students are assessed via:

- A group write-up (all team members collaborate and are marked identically) in the style of Nature Communications, reflecting the submission of a scientific manuscript;

- A group oral presentation to peers and module leads (all team members collaborate, and are marked individually);

- Individual technical and lay abstracts, reflecting the necessity to concisely communicate science to peers and lay audiences, and the required structure of grant proposals.

We designed this strategy both according to constructive alignment principles and to reflect employment - delivering a project as a team, with a mode of individual assessment, and with skills in research and communication (both to peers and lay audiences) jointly tested.

Campbell \& Nehm [Campbell and Nehm, 2013] draw attention to the lack of evidence of reliable, validated assessment in a survey of bioinformatics courses. We ensured that independent assessment of each of these tasks was performed by two experienced and non-collaborating academics, and overseen by an external examiner; we attempted to follow the peer-review model as closely as possible for the write-up assessment while also grading according to defined marking criteria which were visible to the students throughout the project. However, further validation of the assessment structure here will be an important part of future course development.

For (ii), feedback is provided:

- Throughout the project, through regular meetings with the PI. This continual feedback is particularly valuable given Gibbs \& Simpson's [Gibbs and Simpson, 2005] requirements that feedback is appropriate given students' understanding of what they are supposed to be doing. Students evolve through different conceptions of learning [Säljö, 1982] as the project develops; continual feedback in the context of the project ensures compatibility with this evolution. Following [Archer, 2010], this feedback helps the students' understanding without dictating what that understanding should be;

- On the oral presentation, given via Q\&A from peers and module leads, and in detailed comments by a range of 2-4 module leads according to a mark scheme;

- On their write-ups and abstracts, as a set of annotations and notes as in a peer review. 
Regarding feedback as an integral part of the learning process [Cramp, 2011], this strategy helps guide the students' work and develops their experience of a research environment [Evans, 2013]. Following the 'feed-forward' and 'feed-up' strategy [Hounsell et al., 2008], the feedback on their oral presentation and write-ups also prepares them for similar assessment of an individual project later in the course, and is envisaged to help them refine their communication skills more generally for work in academia and broader sectors.

Encouragingly, the groups invariably delivered mature and well-presented research projects, with both written and oral presentations incorporating the ideas from their parallel work on communication and critique. Group write-ups critiqued existing work and provided a well-argued motivating framework for the research question. Results were generally presented in a clear and rhetorically compelling way, although there were several instances where different styling choices could have made deliverables clearer (from small axis labels to different choices of distributional plots). Conclusions were sensible, with little 'overselling' of results to fit a narrative, and discussions often drew on broader literature to frame the outputs and motivate future work. Overall, the group reports read encouragingly like scientific manuscripts ready for submission.

Group oral presentations were also of a high standard. Slides were well designed and the sometimes challenging task of allocating different parts of the delivery to different group members was generally well managed. There was heterogeneity between groups in the amount of time dedicated to motivating and 'setting up' the research question; a common feedback point was that in presenting to an interdisciplinary audience, investing several slides in the broader picture behind the research can help quickly bring everyone up to speed. Q\&A was also generally positive, with student responses to aligned technical questions generally confident and well-reasoned, and with good responses (including some honest answers of 'l'm not sure') to more testing broader questions.

Rather by contrast, the individual written abstracts were more heterogeneous in quality. Technical abstracts were sometimes rather verbose, reading more like the introduction to a paper. Lay abstracts were sometimes very similar copies of the technical version, although there were many more thoughtful instances where well-chosen vocabulary and phrasing was used to simplify the message (Fig. 2). As fitting these individual abstracts to a given audience appears to be a more challenging task, we intend in future to make explicit reference to readability scores like the Flesch-Kincaid grade level [Kincaid et al., 1975] to give a quantitative handle on the complexity of written text.

We have received positive feedback both from students and our external examiner on the multifaceted nature of these assessment principles, suggesting that the this core assessment structure can be retained into the future. However, following the concerns of unequal contributions above, there clearly exists a tension between the pros of representing a genuine group work experience (which always has the risk of unequal contributions) and the cons of perceived unfairness in the resulting assessment. We are exploring suggestions from colleagues and our external examiner about making the structure more robust to situations like student dropouts or 'defectors' in group work.

\section{Conclusions and reflection points}

In the design and maintenance of a bioinformatics course, both general and discipline-specific pedagogical principles can be leveraged. General principles include the rational design of classes, modules, and environments to follow a principled taxonomy of learning [Krathwohl, 2002], assessment structures that 'support worthwhile learning' [Gibbs and Simpson, 2005], and consideration of humanist aspects of teaching style [Rogers et al., 2013] - the latter perhaps being particularly important in a largely connectivist topic [Siemens, 2005] where a social constructivist paradigm can develop and reinforce collaborative skills and make deliverables greater than the sum of their parts [Kim, 2001]. Discipline-specific debate on pedagogical ideas and technology in bioinformatics is somewhat more recent but provides valuable overall 'best practise' ideas [Gnimpieba et al., 2013, Saravanan and Shanmughavel, 2007, Pevzner and Shamir, 2009]. Ongoing monitoring of the societal priorities for technical and scientific skills in bioinformatics, by region and discipline [Hersh, 2008, Koch and Fuellen, 2008, Tastan Bishop et al., 2014], is also valuable in the design and continuing review of learning outcomes that will benefit students not just in the field of bioinformatics but as members of and contributors to broader society.

Siemens [Siemens, 2005] challenges us: 'what adjustments need to made with learning theories when technology performs many of the cognitive operations previously performed by learners?'. Particularly in the connectivist world of bioinformatics, it can potentially be easy to lose a human connection with a class. Indeed, if all knowledge is available via online sources like MOOCs [Ding et al., 2014], what sort of teacher-student relationship is appropriate or needed? Answers to this question are fluid and evolving. Experience through this course suggested that the presence of the instructor in the classroom leads to more rapid and tailored responses to technical questions; improving access across a diverse student group; more opportunities to start and catalyse discussions in social 
learning [Hodkinson and Hodkinson, 2004]; a valuable humanist connection with the class [Rogers et al., 2013] when discussing transferable, teamwork, and communication skills, and particularly ethical topics; and the benefits of personal experience in the field. However, development of bioinformatics teaching must necessitate continual reflection on this question.

Student feedback (Appendix B) has been positive about the aspects of the module discussed above, with some particularly positive notes about increasing accessibility to, and confidence in, a diverse cohort (some examples of comments received were: 'really helped build confidence', 'meant students from a non-computing background could follow along without feeling too lost'). The merit of 'Learning by doing' was also explicitly praised. Positive responses for teaching delivery included finding 'lecturers to be very helpful and willing to give breaks every $45 \mathrm{mins}$ with a chance to ask questions - very helpful', 'Very enthusiastic and knowledgeable', 'Engaging at all times', and the informal but pleasing 'Mr Johnston was very useful and with good vibes'. The intensive nature of information delivery is reflected in 'The speed of speaker is a little bit quickly [sic] for me to follow', suggesting continuing work on this trade-off between enthusiasm and clarity is desirable. These themes begin to answer the earlier question about the merit of the teacher-student relationship in a connectivist topic [Siemens, 2005]. Going forward, we are reviewing structural suggestions that students proposed to facilitate smoother learning and will continue to assess and reflect as the course goes on.

Encouragingly, the inclusion of interdisciplinary concepts in bioinformatics teaching was overwhelmingly positively received by students. Responses to 'Was the content of the lectures and practicals...' involved 13 'highly relevant' (the highest response), 1 'quite useful' (second highest response), 1 split between these two responses, and none for 'limited relevance' or 'useless'. Teaching style was also received positively, in addition to the qualitative points above, with 9 'excellent' and 6 'good' responses and no lower scores. Students have gone on to a diverse range of later employment, mirroring the broad need for bioinformaticians and data scientists in a broad range of fields including a range of 'big' established as well as small to medium sized enterprises in pharmaceuticals, the food and chemical industry, health, and more. Course alumni have gone on to work in the NHS (reflecting an ongoing transformation into the area of genomics and big data), genetics labs and commercial biotechnology companies, within the UK and internationally. Several alumni are working in UK and international forensics, where new genetic testing and data analysis is increasingly demanding bioinformatics skills. More alumni are now in $\mathrm{PhD}$ programmes where bioinformatics, interdisciplinary research skills, and experience with real-world research are in very high demand - destinations include several Russell Group and US universities.

Going forward, we intend to provide increasing numbers of projects with external partners, including industries and institutions. While this expansion will come with increased challenges of monitoring projects, it will progress even further towards the constructive alignment principle of teaching bioinformatics by doing (real-world) bioinformatics in interdisciplinary settings [Biggs, 1996, Pevzner and Shamir, 2009, Goodman and Dekhtyar, 2014]. Connections and experience with industry and other institutions will also provide useful links for potential student destinations after the course. Future axes of expansion of the course include geography (expanding to international campuses), content (including reviewing integration with an overlapping MSc Health Data Science) and format (including expansion into distance learning and apprenticeship models). Further parallel course design will development and implement an equivalent module to that outlined here in these more diverse settings.

To conclude, we hope that this report has served to argue the value of including interdisciplinary and transferable concepts in bioinformatics education, and indeed that the field can provide excellent opportunities to develop research-led, group learning [Goodman and Dekhtyar, 2014, Magana et al., 2014] and expansive discussion on important ethical issues in bioinformatics [Taneri, 2011]. The diversity of bioinformatics student cohorts can pose challenges (which we can combat through 'diversity-addressing' approaches) but also present opportunities for constructive learning and group research (which we can harness using 'diversity-exploiting' approaches) that prepares students for a wide range of future activities in society.

\section{Acknowledgements}

IGJ is grateful to the Educational Development team at the University of Birmingham for their support in preparing this report. The authors thank the module leads for supporting the development and delivery of this course.

This report received ethical approval from the Science, Technology, Engineering and Mathematics Ethical Review Committee at the University of Birmingham. 


\section{References}

[fao, 2019] (2019). Fao: Food loss and food waste.

[Abolins et al., 2017] Abolins, S., King, E. C., Lazarou, L., Weldon, L., Hughes, L., Drescher, P., Raynes, J. G., Hafalla, J. C., Viney, M. E., and Riley, E. M. (2017). The comparative immunology of wild and laboratory mice, mus musculus domesticus. Nature communications, 8:14811.

[Adami et al., 2000] Adami, C., Ofria, C., and Collier, T. C. (2000). Evolution of biological complexity. Proceedings of the National Academy of Sciences, 97(9):4463-4468.

[Afgan et al., 2015] Afgan, E., Sloggett, C., Goonasekera, N., Makunin, I., Benson, D., Crowe, M., Gladman, S., Kowsar, Y., Pheasant, M., Horst, R., et al. (2015). Genomics virtual laboratory: a practical bioinformatics workbench for the cloud. PloS one, 10(10):e0140829.

[Ai et al., 2012] Ai, Y.-C., Firth, N., and Jermiin, L. (2012). Teaching bioinformatics: A student-centred and problem based approach. International Journal of Innovation in Science and Mathematics Education (formerly CALlaborate International), 10(1).

[Alon, 2009] Alon, U. (2009). How to give a good talk. Molecular cell, 36(2):165-167.

[Altman, 1998] Altman, R. B. (1998). A curriculum for bioinformatics: the time is ripe. Bioinformatics (Oxford, England), 14(7):549-550.

[Amer, 2017] Amer, S. (2017). Ethical concerns regarding the use of bioinformatics and computational genomics. In Proceedings of the International Conference on e-Learning, e-Business, Enterprise Information Systems, and e-Government (EEE), pages 58-62. The Steering Committee of The World Congress in Computer Science, Computer .

[Archer, 2010] Archer, J. C. (2010). State of the science in health professional education: effective feedback. Medical education, 44(1):101-108.

[Beauchamp et al., 2001] Beauchamp, T. L., Childress, J. F., et al. (2001). Principles of biomedical ethics. Oxford University Press, USA.

[Begley and Ellis, 2012] Begley, C. G. and Ellis, L. M. (2012). Drug development: Raise standards for preclinical cancer research. Nature, 483(7391):531.

[Belbin, 2012] Belbin, R. M. (2012). Team roles at work. Routledge.

[Biggs, 1996] Biggs, J. (1996). Enhancing teaching through constructive alignment. Higher education, 32(3):347364.

[Bloom et al., 1956] Bloom, B. S. et al. (1956). Taxonomy of educational objectives. vol. 1: Cognitive domain. New York: McKay, pages 20-24.

[Borsuk et al., 2009] Borsuk, R. M., Aarssen, L. W., Budden, A. E., Koricheva, J., Leimu, R., Tregenza, T., and Lortie, C. J. (2009). To name or not to name: The effect of changing author gender on peer review. BioScience, 59(11):985-989.

[Brown, 2016] Brown, J. A. (2016). Evaluating the effectiveness of a practical inquiry-based learning bioinformatics module on undergraduate student engagement and applied skills. Biochemistry and molecular biology education, 44(3):304-313.

[Bustamante et al., 2011] Bustamante, C. D., Francisco, M., and Burchard, E. G. (2011). Genomics for the world. Nature, 475(7355):163.

[Campbell and Nehm, 2013] Campbell, C. E. and Nehm, R. H. (2013). A critical analysis of assessment quality in genomics and bioinformatics education research. CBELife Sciences Education, 12(3):530-541.

[Cattley and Arthur, 2007] Cattley, S. and Arthur, J. W. (2007). Biomanager: the use of a bioinformatics web application as a teaching tool in undergraduate bioinformatics training. Briefings in bioinformatics, 8(6):457465. 
[Collaboration et al., 2015] Collaboration, O. S. et al. (2015). Estimating the reproducibility of psychological science. Science, 349(6251):aac4716.

[Collin and Valleala, 2005] Collin, K. and Valleala, U. M. (2005). Interaction among employees: how does learning take place in the social communities of the workplace and how might such learning be supervised? Journal of Education and Work, 18(4):401-420.

[Conway, 1970] Conway, J. (1970). The game of life. Scientific American, 223(4):4.

[Cramp, 2011] Cramp, A. (2011). Developing first-year engagement with written feedback. Active Learning in Higher Education, 12(2):113-124.

[Cummings and Temple, 2010] Cummings, M. P. and Temple, G. G. (2010). Broader incorporation of bioinformatics in education: opportunities and challenges. Briefings in bioinformatics, 11(6):537-543.

[Dahlö et al., 2015] Dahlö, M., Haziza, F., Kallio, A., Korpelainen, E., Bongcam-Rudloff, E., and Spjuth, O. (2015). Biolmg. org: A catalog of virtual machine images for the life sciences. Bioinformatics and Biology insights, 9:BBI-S28636.

[Ding et al., 2014] Ding, Y., Wang, M., He, Y., Ye, A. Y., Yang, X., Liu, F., Meng, Y., Gao, G., and Wei, L. (2014). bioinformatics: Introduction and methods, a bilingual massive open online course (mooc) as a new example for global bioinformatics education. PLoS computational biology, 10(12):e1003955.

[Editorial, 2016] Editorial, N. (2016). Rethink the links between genes and disease.

[Edmonds, 1995] Edmonds, B. (1995). What is complexity?-the philosophy of complexity per se with application to some examples in evolution. In The evolution of complexity. Kluwer, Dordrecht.

[Evans, 2013] Evans, C. (2013). Making sense of assessment feedback in higher education. Review of educational research, 83(1):70-120.

[Fulekar, 2009] Fulekar, M. (2009). Bioinformatics: applications in life and environmental sciences. Springer Science \& Business Media.

[Furge et al., 2009] Furge, L. L., Stevens-Truss, R., Moore, D. B., and Langeland, J. A. (2009). Vertical and horizontal integration of bioinformatics education: a modular, interdisciplinary approach. Biochemistry and Molecular Biology Education, 37(1):26-36.

[Gibbs and Simpson, 2005] Gibbs, G. and Simpson, C. (2005). Conditions under which assessment supports students learning. Learning and teaching in higher education, (1):3-31.

[Gnimpieba et al., 2013] Gnimpieba, E. Z., Jennewein, D., Fuhrman, L., and Lushbough, C. M. (2013). Bioinformatics knowledge transmission (training, learning, and teaching): overview and flexible comparison of computer based training approaches. arXiv preprint arXiv:1310.8383.

[Gonzalez, 2004] Gonzalez, C. (2004). The role of blended learning in the world of technology. Retrieved December, 10:2004.

[Goodman and Dekhtyar, 2014] Goodman, A. L. and Dekhtyar, A. (2014). Teaching bioinformatics in concert. PLoS computational biology, 10(11):e1003896.

[Goodman and Cava, 2008] Goodman, K. W. and Cava, A. (2008). Bioethics, business ethics, and science: Bioinformatics and the future of healthcare. Cambridge Quarterly of Healthcare Ethics, 17(4):361-372.

[Greenbaum et al., 2011] Greenbaum, D., Sboner, A., Mu, X. J., and Gerstein, M. (2011). Genomics and privacy: implications of the new reality of closed data for the field. PLoS Computational Biology, 7(12):e1002278.

[Hack and Kendall, 2005a] Hack, C. and Kendall, G. (2005a). Bioinformatics: Current practice and future challenges for life science education. Biochemistry and Molecular Biology Education, 33(2):82-85.

[Hack and Kendall, 2005b] Hack, C. and Kendall, G. (2005b). Bioinformatics education in the uk: Are we educating scientists or training technicians? Bioscience Bulletin, 15:10. 
[Hersh, 2008] Hersh, W. (2008). Health and biomedical informatics: opportunities and challenges for a twenty-first century profession and its education. Yearbook of medical informatics, 17(01):157-164.

[Hodkinson and Hodkinson, 2004] Hodkinson, H. and Hodkinson, P. (2004). Rethinking the concept of community of practice in relation to schoolteachers workplace learning. International journal of training and development, $8(1): 21-31$.

[Hodkinson et al., 2004] Hodkinson, P., Biesta, G., and James, D. (2004). Towards a cultural theory of collegebased learning. In Manchester Annual Conference of the British Educational Research Association.

[Homer et al., 2008] Homer, N., Szelinger, S., Redman, M., Duggan, D., Tembe, W., Muehling, J., Pearson, J. V., Stephan, D. A., Nelson, S. F., and Craig, D. W. (2008). Resolving individuals contributing trace amounts of dna to highly complex mixtures using high-density snp genotyping microarrays. PLoS genetics, 4(8):e1000167.

[Hounsell et al., 2008] Hounsell, D., McCune, V., Hounsell, J., and Litjens, J. (2008). The quality of guidance and feedback to students. Higher Education Research \& Development, 27(1):55-67.

[loannidis, 2005] loannidis, J. P. (2005). Why most published research findings are false. PLoS medicine, 2(8):e124.

[Johnston, 1997] Johnston, S. (1997). Using peer support: Implications for student and lecturer. ASPECTS OF EDUCATIONAL AND TRAINING TECHNOLOGY, 29:69-76.

[Kikin et al., 2006] Kikin, O., D’Antonio, L., and Bagga, P. S. (2006). Qgrs mapper: a web-based server for predicting g-quadruplexes in nucleotide sequences. Nucleic acids research, 34(suppl_2):W676-W682.

[Kim, 2001] Kim, B. (2001). Social constructivism. Emerging perspectives on learning, teaching, and technology, $1(1): 16$.

[Kincaid et al., 1975] Kincaid, J. P., Fishburne Jr, R. P., Rogers, R. L., and Chissom, B. S. (1975). Derivation of new readability formulas (automated readability index, fog count and flesch reading ease formula) for navy enlisted personnel. Research Branch Report 8-75, Millington, TN: Naval Technical Training, U. S. Naval Air Station, Memphis, TN.

[Koch and Fuellen, 2008] Koch, I. and Fuellen, G. (2008). A review of bioinformatics education in germany. Briefings in bioinformatics, 9(3):232-242.

[Krathwohl, 2002] Krathwohl, D. R. (2002). A revision of bloom's taxonomy: An overview. Theory into practice, 41(4):212-218.

[Kuhn, 2012] Kuhn, T. S. (2012). The structure of scientific revolutions. University of Chicago press.

[Lambert and Black, 2012] Lambert, C. G. and Black, L. J. (2012). Learning from our gwas mistakes: from experimental design to scientific method. Biostatistics, 13(2):195-203.

[Lathan et al., 2002] Lathan, C. E., Tracey, M. R., Sebrechts, M. M., Clawson, D. M., and Higgins, G. A. (2002). Using virtual environments as training simulators: Measuring transfer. Handbook of virtual environments: Design, implementation, and applications, pages 403-414.

[Lek et al., 2016] Lek, M., Karczewski, K. J., Minikel, E. V., Samocha, K. E., Banks, E., Fennell, T., ODonnellLuria, A. H., Ware, J. S., Hill, A. J., Cummings, B. B., et al. (2016). Analysis of protein-coding genetic variation in 60,706 humans. Nature, 536(7616):285.

[Levy, 1993] Levy, S. (1993). Artificial life: A report from the frontier where computers meet biology. Random House Inc.

[Luscombe et al., 2001] Luscombe, N. M., Greenbaum, D., and Gerstein, M. (2001). What is bioinformatics? a proposed definition and overview of the field. Methods of information in medicine, 40(04):346-358.

[Magana et al., 2014] Magana, A. J., Taleyarkhan, M., Alvarado, D. R., Kane, M., Springer, J., and Clase, K. (2014). A survey of scholarly literature describing the field of bioinformatics education and bioinformatics educational research. CBELife Sciences Education, 13(4):607-623. 
[Markowetz, 2017] Markowetz, F. (2017). All biology is computational biology. PLoS biology, 15(3):e2002050.

[McKinney, 2010] McKinney, K. (2010). Active learning. illinois state university. Center for Teaching, Learning \& Technology.

[Mensh and Kording, 2017] Mensh, B. and Kording, K. (2017). Ten simple rules for structuring papers.

[Merrill, 2002] Merrill, M. D. (2002). First principles of instruction. Educational technology research and development, 50(3):43-59.

[Michaelsen et al., 2002] Michaelsen, L. K., Stanley, C., and Porter, M. (2002). Team learning in large classes. Engaging large classes: strategies and techniques for college faculty, 27:67.

[Ndebele and Musesengwa, 2008] Ndebele, P. and Musesengwa, R. (2008). Will developing countries benefit from their participation in genetics research? Malawi Medical Journal, 20(2):67-69.

[Novembre et al., 2008] Novembre, J., Johnson, T., Bryc, K., Kutalik, Z., Boyko, A. R., Auton, A., Indap, A., King, K. S., Bergmann, S., Nelson, M. R., et al. (2008). Genes mirror geography within europe. Nature, 456(7218):98.

[Ofria and Wilke, 2004] Ofria, C. and Wilke, C. O. (2004). Avida: A software platform for research in computational evolutionary biology. Artificial life, 10(2):191-229.

[Pearce, 1995] Pearce, D. (1995). Hedonistic imperative. David Pearce.

[Pevzner and Shamir, 2009] Pevzner, P. and Shamir, R. (2009). Computing has changed biologybiology education must catch up. Science, 325(5940):541-542.

[Popejoy and Fullerton, 2016] Popejoy, A. B. and Fullerton, S. M. (2016). Genomics is failing on diversity. Nature News, 538(7624):161.

[Prinz et al., 2011] Prinz, F., Schlange, T., and Asadullah, K. (2011). Believe it or not: how much can we rely on published data on potential drug targets? Nature reviews Drug discovery, 10(9):712.

[Prusinkiewicz et al., 1999] Prusinkiewicz, P., Karwowski, R., Měch, R., and Hanan, J. (1999). L-studio/cpfg: a software system for modeling plants. In International Workshop on Applications of Graph Transformations with Industrial Relevance, pages 457-464. Springer.

[R Core Team, 2015] R Core Team (2015). R: A Language and Environment for Statistical Computing. R Foundation for Statistical Computing, Vienna, Austria.

[Ramsden, 2003] Ramsden, P. (2003). Learning to teach in higher education. Routledge.

[Ranganathan, 2005] Ranganathan, S. (2005). Bioinformatics educationperspectives and challenges. PLoS computational biology, 1(6):e52.

[Ray, 1991] Ray, T. S. (1991). An approach to the synthesis of life. Artificial life II, pages 371-408.

[Rogers et al., 2013] Rogers, C. R., Lyon, H. C., and Tausch, R. (2013). On becoming an effective teacher: Person-centered teaching, psychology, philosophy, and dialogues with Carl R. Rogers and Harold Lyon. Routledge.

[Salazar, 2018] Salazar, M. (2018). Brainstorming for innovation in team science.

[Salazar et al., 2012] Salazar, M. R., Lant, T. K., Fiore, S. M., and Salas, E. (2012). Facilitating innovation in diverse science teams through integrative capacity. Small Group Research, 43(5):527-558.

[Säljö, 1982] Säljö, R. (1982). Learning and understanding: A study of differences in constructing meaning from a text, volume 41. Humanities Pr.

[Saravanan and Shanmughavel, 2007] Saravanan, V. and Shanmughavel, P. (2007). E-learning as a new tool in bioinformatics teaching. Bioinformation, 2(3):83.

[Schneider et al., 2011] Schneider, M. V., Walter, P., Blatter, M.-C., Watson, J., Brazas, M. D., Rother, K., Budd, A., Via, A., van Gelder, C. W., Jacob, J., et al. (2011). Bioinformatics training network (btn): a community resource for bioinformatics trainers. Briefings in bioinformatics, 13(3):383-389. 
[Schroeder, 2007] Schroeder, D. (2007). Benefit sharing: its time for a definition. Journal of medical ethics, 33(4):205-209.

[Schüklenk and Kleinsmidt, 2006] Schüklenk, U. and Kleinsmidt, A. (2006). North-south benefit sharing arrangements in bioprospecting and genetic research: a critical ethical and legal analysis. Developing World Bioethics, 6(3):122-134.

[Seife, 2013] Seife, C. (2013). 23andme is terrifying, but not for the reasons the fda thinks. Scientific American, 27(11).

[Siemens, 2005] Siemens, G. (2005). Connectivism: Learning as network-creation. ASTD Learning News, 10(1):1-28.

[Sorge et al., 2014] Sorge, R. E., Martin, L. J., Isbester, K. A., Sotocinal, S. G., Rosen, S., Tuttle, A. H., Wieskopf, J. S., Acland, E. L., Dokova, A., Kadoura, B., et al. (2014). Olfactory exposure to males, including men, causes stress and related analgesia in rodents. Nature methods, 11(6):629.

[St Clair and Visick, 2010] St Clair, C. and Visick, J. (2010). Exploring Bioinformatics: A Project-based Approach. Jones and Bartlett Publishers. USA.

[Taneri, 2011] Taneri, B. (2011). Is there room for ethics within bioinformatics education? Journal of Computational Biology, 18(7):907-916.

[Tastan Bishop et al., 2014] Tastan Bishop, Ö., Adebiyi, E. F., Alzohairy, A. M., Everett, D., Ghedira, K., Ghouila, A., Kumuthini, J., Mulder, N. J., Panji, S., Patterton, H.-G., et al. (2014). Bioinformatics educationperspectives and challenges out of africa. Briefings in bioinformatics, 16(2):355-364.

[Tomlinson, 2008] Tomlinson, M. (2008). the degree is not enough: students perceptions of the role of higher education credentials for graduate work and employability. British journal of sociology of education, 29(1):4961.

[Von Neumann et al., 1966] Von Neumann, J., Burks, A. W., et al. (1966). Theory of self-reproducing automata. IEEE Transactions on Neural Networks, 5(1):3-14.

[Warhurst, 2008] Warhurst, R. P. (2008). cigars on the flight-deck: new lecturers participatory learning within workplace communities of practice. Studies in higher education, 33(4):453-467.

[Welch et al., 2014] Welch, L., Lewitter, F., Schwartz, R., Brooksbank, C., Radivojac, P., Gaeta, B., and Schneider, M. V. (2014). Bioinformatics curriculum guidelines: toward a definition of core competencies. PLOS computational biology, 10(3):e1003496.

[Wickham, 2009] Wickham, H. (2009). ggplot2: Elegant Graphics for Data Analysis. Springer-Verlag New York.

[Wiggins et al., 2005] Wiggins, G., Wiggins, G. P., and McTighe, J. (2005). Understanding by design. Ascd.

[Yazedjian and Kolkhorst, 2007] Yazedjian, A. and Kolkhorst, B. B. (2007). Implementing small-group activities in large lecture classes. College Teaching, 55(4):164-169.

\section{Appendix A - QAA descriptors}

Corresponding elements of designed module outcomes (Table 1) are given in parentheses.

The general QAA descriptors at this level involve (paraphrasing):

- Systematic understanding of knowledge and critical awareness of forefront of discipline [(b), (c), (e)]

- Comprehensive understanding of techniques [(b), (c), (d), (e)]

- Original applications of knowledge [(d)]

- Critically evaluate current research and methodologies [(e)]

- Systematically and creatively deal with complex issues [(d)]

- Self-direction and originality [entire module]

- Employment and personal skills [entire module]

On graduating with an honours degree in biosciences, graduates will have the following core knowledge, understanding and skills: 
- experience and competence in a broad range of appropriate practical techniques and skills relevant to the biosciences including data collection, analysis and interpretation of those data, and testing of hypotheses and the ability to place the work in context and to suggest lines of further investigation [mainly (d)]

- the ability to explain biological phenomena at a variety of levels (from molecular to ecological systems) and how evolutionary theory is relevant to their area of study [(c), (e), (f)]

- the ability to plan, execute and present a piece of hypothesis-driven work within a supported framework in which qualities such as time management, problem solving, and independence are evident [entire module]

- the ability to access and evaluate bioscience information from a variety of sources and to communicate the principles both orally and in writing in a way that is organised and topical, and recognises the limits of current hypotheses [(b), (e), (f)]

- an appreciation of ethical issues and how they underpin professional integrity and standards [(e)]

- an appreciation of the impact on society of advances in the biosciences [(e)]

- the ability to record data accurately, and to carry out basic manipulation of data (including qualitative data and statistical analysis, when appropriate) [(c), (d)]

- an understanding of the use of bioinformatics approaches in the analysis of large datasets [entire module] On graduating with an honours degree in biosciences, in addition to demonstrating the core knowledge, understanding and skills specified in the threshold standard, a graduate will be able to:

- plan, execute and present an independent piece of work, in which qualities such as time management, problem solving and independence are evident, as well as interpretation and critical awareness of the quality of evidence [individual assessment; also covered in individual projects in a parallel module]

- construct reasoned arguments to support their position on the ethical and social impact of advances in the biosciences [(e), (f)]

- demonstrate a secure and accurate understanding of the explanation of biological phenomena at a variety of levels (from molecular to ecological systems) and explain the relationship of evolutionary theory to their area of study [(b), (c), (e)]

- apply relevant advanced numerical skills to biological data [(d)]

- communicate science to peers and non-scientists [(f)]

- demonstrate well developed strategies for updating, maintaining and enhancing their knowledge of the biosciences, including cross-disciplinary awareness access bioscience databases and use appropriate selection criteria to mine, manipulate and interpret data. [entire module]

\section{Appendix B - Student feedback}

General positive observations:

- 'Learning by doing' [Warhurst, 2008] explicitly praised (I.1.C.) with many other positive notes about the combination of theory and practice (I.2.; I.3.; I.4; I.5; etc).

- Step-by-step approach helps students from different backgrounds avoid feeling lost, increasing accessibility (I.1.a.; UK PSF V1, V2).

- Well-spaced breaks (which aren't strictly aligned with hourly schedule) praised for allowing consolidation and troubleshooting (I.7.b.; I.13.; I.16.c.)

General notes for improvement:

- Earlier release of material and/or reading would be useful (II.1; II.13.b.; III.3.a.)

- Spreading out content and consolidating over multiple days would have helped (even without lecturer contact) (II.3.; II.5.; II.6.; II.11.)

- More time on content / ensure all content is completed (II.4.; II.7.; II.13.a.; III.5.)

Numerical labels below reference individual student responses.

I. What aspects of the teaching best helped your learning?

- I.1.a. A step by step approach to introducing new software and the fundamentals of programming, meant students from a non-computing background could follow along without feeling too lost.

- I.1.b. Learning command line then Python and finally applying it to $R$ really helped build confidence with using different software/languages.

- I.1.c. Learning by "doing". Given the opportunity to work out a pipeline before being shown solutions helped think creatively about problem solving.

- I.2. They showed the examples and asked to do some practices which is a good way to understand what I have learnt 
- I.3.a. Lecturers been very understanding and helpful when teaching us. Exercises provided very helpful at getting us to practise.

- I.3.b. Practising better than learning by theory.

- I.4. The examples and interactive aspect of the lectures.

- I.5. Letting us do the activities in the [programming] sessions to reinforce our learning

- I.6. This module has been highly relevant as an introduction to the course, however I have found it very full on with a lot of new information in a short space of time. [...] I found the lecturers to be very helpful and willing to give breaks every 45 mins with a chance to ask questions - very helpful.

- I.7.a. Working through the code as a class when learning Python was highly effective.

- I.7.b. Frequent short breaks after learning new concepts made it easier to consolidate learning.

- I.8.a. Many practical examples

- I.8.b. The information found on Canvas

- 1.9. Practising different aspects of the code that we are learning

- I.10. Time for exercises where the lecturer was able to solve our questions individually

- I.11. The practical sessions were the most helpful. However, the theoretical parts were also very useful but a little intense

- I.13. Frequent breaks for questions \& troubleshooting

- I.14.a. Practical mixed with theory (really good).

- I.14.b. Python lectures were good though I found them too slow

- I.15. Lots of helpful worked examples so we can apply things we have learnt, right away

- I.16.a. Very enthusiastic and knowledgable

- I.16.b. Engaging at all times

- I.16.c. Good well spaced breaks

- I.16.d. Good amount of content

II. What could have been done differently to help you learn more effectively?

- II.1. Earlier release of pre-course material! It would really help if applicants could have a chance to look [at] command line, R, Python before the course started.

- II.2. The speed of speaker is a little bit quickly for me to follow

- II.3. Spreading out the teaching content over the whole 2 weeks - we had 4 days off which could've been used for applying what we have learnt with help available if we needed.

- II.4. If there was more time to go through the introductions.

- II.5. Provide a tutorial a day after learning a coding language. This will help us understand and remember concepts, more than having just a lecture followed with [...]?

- II.6. [I wonder if it might be useful to have 1 day of lectures followed by one day of self-study, spread more over the first 2 weeks so that there is time to make sense of each topic straight after being taught it.]

- II.7.a. Ensure all scheduled content is completed.

- II.7.b. It is slightly overwhelming knowing that some key concepts were not [covered] due to time constraints.

- II.9. Some deeper explanations of some of the "R" code with examples

- II.10. More examples for the codes on the lecture handouts

- II.11. Split out the lectures, in order to some parts would be more understanded

- II.12. More time for practice

- II.13.a. More time on each system - we ran out of time with each one.

- II.13.b. Some recommended reading to introduce concepts could cut down time spent just listening about them instead of practice.

- II.13.c. Generally, I felt there was a steep step up between the introduction to concepts \& using them in practice

- II.16. More on Python and self created functions

III. Any other comments you wish to make:

- III.1. Really liked the xkcd comics!

- III.3.a. We were only sent the reading 1 week before the course started - it had lots to read on it, so would have been much better to get this earlier so we would have time to do more.

- III.3.b. Lecturers so far have been great :D

- III.5. For lecturers to have more time to teach as all 3 coding languages weren't completed due to running out of time.

- III.11. Mr Johnston was very useful and with good vibes 


\section{Appendix C - Online resources}

Artificial life:

- Video of emergence of self-reproducing 'evoloop' automata https : //www . youtube. com/watch?v=5IpK5meen28

- Prime number calculation by automata in Game of Life https ://www . youtube . com/watch?v=68nEX5CEmZE Regex Golf:

- http://regex.alf.nu/

Science Heroes:

- http://www.scienceheroes.com

xkcd comics:

- 'Biology is largely solved' https://xkcd.com/1605/

- Regular expressions https://xkcd.com/208/

- tar https://xkcd.com/1168/

- p-values https://xkcd.com/882/

- Linux cheat sheet https://store.xkcd.com/products/linux-cheat-shirt

- Frequentist versus Bayesian statistics https://xkcd.com/1132/

- Dubious correlations https://xkcd.com/1725/

Readability:

- Readability Analyzer https://datayze.com/readability-analyzer.php

Science, ethics, and society:

- Causes of death and disability http://www.who.int/gho/mortality_burden_disease/causes_death/top_ $10 /$ en/

- Health and health spending https://ourworldindata.org/health-meta/

- UK cancer spending https://www.statista.com/chart/2825/uk-cancer-spending/

- Public health and smoking https://www.cdc.gov/cancer/lung/basic_info/risk_factors.htm and nutrition http://www. who.int/mediacentre/factsheets/malnutrition/en/

- Food waste http://thebrazilbusiness.com/article/food-waste-sector-in-brazil

- FAO Food Loss and Waste http://www.fao.org/food-loss-and-food-waste/en/

- Coverage of ExAC study findings https ://www . nature.com/news/rethink-the-links-between-genes-and-disease-1 20771

- 23AndMe data use https://www.scientificamerican.com/article/23andme-is-terrifying-but-not-for-the-reas 